The same number of optimized parameters scheme for determining intermolecular interaction energies

Kasper Kristensen’, Patrick Ettenhuber, Janus Juul Eriksen, Frank Jensen, and Poul Jørgensen

Citation: The Journal of Chemical Physics 142, 114116 (2015); doi: 10.1063/1.4915141

View online: http://dx.doi.org/10.1063/1.4915141

View Table of Contents: http://aip.scitation.org/toc/jcp/142/11

Published by the American Institute of Physics

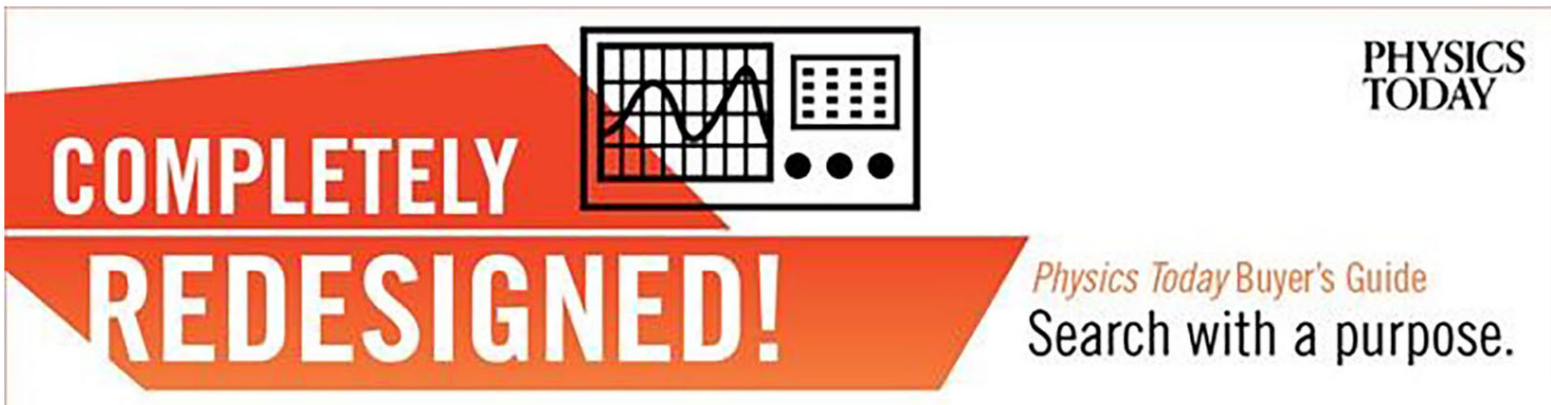




\title{
The same number of optimized parameters scheme for determining intermolecular interaction energies
}

\author{
Kasper Kristensen, ${ }^{1, a)}$ Patrick Ettenhuber,${ }^{1}$ Janus Juul Eriksen, ${ }^{1}$ Frank Jensen, ${ }^{2}$ and \\ Poul Jørgensen ${ }^{1}$ \\ ${ }^{1}$ Department of Chemistry, qLEAP Center for Theoretical Chemistry, Aarhus University, Langelandsgade 140, \\ DK-8000 Aarhus C, Denmark \\ ${ }^{2}$ Department of Chemistry, Aarhus University, Langelandsgade 140, DK-8000 Aarhus C, Denmark
}

(Received 15 December 2014; accepted 4 March 2015; published online 20 March 2015)

\begin{abstract}
We propose the Same Number Of Optimized Parameters (SNOOP) scheme as an alternative to the counterpoise method for treating basis set superposition errors in calculations of intermolecular interaction energies. The key point of the SNOOP scheme is to enforce that the number of optimized wave function parameters for the noninteracting system is the same as for the interacting system. This ensures a delicate balance between the quality of the monomer and dimer finite basis set calculations. We compare the SNOOP scheme to the uncorrected and counterpoise schemes theoretically as well as numerically. Numerical results for second-order Møller-Plesset perturbation theory (MP2) and coupled-cluster with single, double, and approximate triple excitations $(\operatorname{CCSD}(\mathrm{T}))$ show that the SNOOP scheme in general outperforms the uncorrected and counterpoise approaches. Furthermore, we show that SNOOP interaction energies calculated using a given basis set are of similar quality as those determined by basis set extrapolation of counterpoise-corrected results obtained at a similar computational cost. @ 2015 AIP Publishing LLC. [http://dx.doi.org/10.1063/1.4915141]
\end{abstract}

\section{INTRODUCTION}

The determination of intermolecular interaction energies remains one of the central tasks of computational chemistry with applications in diverse fields such as molecular biology and material sciences. The interaction energy of two monomers $A$ and $B, \Delta E_{A B}$, is defined as the difference between the energy of the interacting dimer system $A B$ and a system of noninteracting monomers $A+B$ infinitely far apart. Standard quantum chemistry programs use atom-centered basis sets, and it is well-known that $\Delta E_{A B}$ obtained by a straightforward (uncorrected) calculation is usually too negative. This occurs because the energy is lowered as the monomers approach each other due to (i) the physical interaction between the monomers and (ii) because monomer $A$ in the $A B$ complex can lower its energy by using the atomic basis functions of monomer $B$ (and vice versa). Effect (i) is the desired interaction component, while effect (ii) is an artifact of using an incomplete oneelectron basis usually referred to as the basis set superposition error (BSSE). ${ }^{1}$ The BSSE disappears in the limit of a complete basis set (CBS), but in practical calculations it is not possible to separate the two effects.

The most commonly used approach for approximately correcting for the BSSE is the counterpoise (CP) approach by Boys and Bernardi. ${ }^{2}$ In this approach, the isolated monomer $A$ calculation is carried out using the basis functions on monomer $A$ as well as the basis functions of monomer $B$ positioned at the dimer geometry (and vice versa). The BSSE is then defined as the difference between the CP-corrected monomer energies and the uncorrected monomer energies. However, while the CP

\footnotetext{
a)Electronic mail: kasperk@chem.au.dk
}

approach has been used in numerous applications, its justification has also been debated extensively in the literature. ${ }^{3-16}$

An alternative to the standard CP approach ${ }^{2}$ is the socalled virtual CP (VCP) approach ${ }^{3,4}$ where the virtual space in each of the monomer calculations has the same dimension as the virtual dimer space. Scaled CP corrections have also been used, ${ }^{17-19}$ and the semi-empirical geometrical CP approach ${ }^{20}$ has recently been suggested as a pragmatic approach to treat the BSSE for large molecular systems. Strategies where the BSSE is eliminated a priori have also been proposed, such as symmetry-adapted perturbation theory, ${ }^{21,22}$ the chemical Hamiltonian approach, ${ }^{23,24}$ the strictly monomer-molecular orbital approach, ${ }^{25}$ and the constrained dimer function approach. ${ }^{26}$ Despite this manifold of alternatives, most applications continue to use the CP procedure, and for this reason, the focus in the present work will be on uncorrected and CPcorrected interaction energies.

Uncorrected interaction energies will most often be too attractive when calculated with a finite basis set, while CPcorrected interaction energies will not be attractive enough. We propose that this situation may be understood and remedied by analyzing the number of wave function parameters in the interacting $A B$ and the noninteracting $A+B$ systems. It turns out that the number of wave function parameters is smaller/larger for the $A+B$ system than for the $A B$ system when the uncorrected/CP-corrected approaches are used. We therefore propose the Same Number Of Optimized Parameters (SNOOP) method where we impose that the number of optimized wave function parameters remains the same for the noninteracting and interacting systems. The concept of an identical number of parameters is applicable to both the Hartree-Fock (HF) and correlated models. In the present work, 
we focus on the coupled-cluster (CC) hierarchy and present numerical results for the second-order Møller-Plesset perturbation theory (MP2) ${ }^{27}$ and CC with single, double, and approximate triple excitations $(\mathrm{CCSD}(\mathrm{T}))^{28}$ models.

The uncorrected, CP, and SNOOP schemes all use the same dimer energy, while the monomer calculations differ. The inherent parameter balance implies that the SNOOP interaction energy by construction will be sandwiched between the uncorrected and $\mathrm{CP}$-corrected interaction energies. This is confirmed by numerical results that show how the SNOOP scheme in general outperforms the uncorrected and CP approaches, which tend to over- and underestimate the magnitude of the interaction energy, respectively.

The outline of the paper is as follows. In Sec. II, we discuss the uncorrected and CP approaches for determining intermolecular interaction energies, while the SNOOP scheme is presented in Sec. III. Section IV contains computational details, and uncorrected, CP, and SNOOP interaction energies at the MP2 and CCSD(T) levels for a selection of test molecules are compared in Sec. V. Finally, Sec. VI contains some concluding remarks and perspectives.

\section{CONVENTIONAL DETERMINATION OF INTERACTION ENERGIES}

Consider two isolated monomers $A$ and $B$ with energies $E_{A}$ and $E_{B}$. The interaction energy of the $A B$ dimer, $\Delta E_{A B}$, is defined as the difference between the total energy of $A B, E_{A B}$, and the total energy of the noninteracting monomers, $E_{A}+E_{B}$, i.e.,

$$
\Delta E_{A B}=E_{A B}-E_{A}-E_{B} .
$$

To simplify the following analysis, we consider only the electronic contribution to the interaction energy. We thus do not consider changes in the zero point vibrational energy or the geometry relaxation of the monomer in the interacting system. In the following, the noninteracting monomers $A$ and $B$ will be collectively referred to as $A+B$.

The practical determination of $\Delta E_{A B}$ involves three separate calculations-one for each of the $A B, A$, and $B$ systems. In a conventional quantum chemical calculation, atom-centered basis functions (atomic orbitals, AOs) are used, and each monomer thus has its own set of AOs, while the $A B$ calculation uses AOs on both systems. When the calculation of $E_{A}\left(E_{B}\right)$ involves only $\mathrm{AOs}$ on monomer $A(B)$ we will refer to the resulting $\Delta E_{A B}$ as the uncorrected interaction energy. Using the notation $\{\ldots\}$ to emphasize the basis set employed in each calculation, the uncorrected (uc) interaction energy is defined as

$$
\Delta E_{A B}^{\mathrm{uc}}=E_{A B}^{\{A B\}}-E_{A}^{\{A\}}-E_{B}^{\{B\}} .
$$

The dimer energy $E_{A B}$ equals the sum of the monomer energies when $A$ and $B$ are infinitely far apart, while it changes as a result of the physical interactions between $A$ and $B$ when $A$ and $B$ approach each other. However, for finite basis sets, the use of Eq. (2) leads to an additional artificial attraction between $A$ and $B$ related to the incompleteness of the monomer basis sets. This happens because system $A$ in the dimer calculation may lower its energy by using the AOs of system $B$ and vice versa. This effect is referred to as the BSSE, ${ }^{1}$ and it implies that interaction energies determined in a finite basis calculation using Eq. (2) will usually be too attractive. The BSSE is not present in the limit where $A$ and $B$ are infinitely far apart or in the hypothetical limit where $A$ and $B$ each possesses a CBS.

The standard approach for estimating the BSSE is the CP correction $^{2}$ where the monomer calculations employ the full $A B$ basis, i.e.,

$$
\Delta E_{A B}^{\mathrm{cp}}=E_{A B}^{\{A B\}}-E_{A}^{\{A B\}}-E_{B}^{\{A B\}} .
$$

For variational wave function models, this will lower the energies of the monomers compared to Eq. (2),

$$
E_{A}^{\{A B\}}+E_{B}^{\{A B\}}<E_{A}^{\{A\}}+E_{B}^{\{B\}} .
$$

Although Eq. (4) is only strictly valid for variational theories, it is usually also observed for nonvariational theories. Since the $A B$ dimer energy is the same in Eqs. (2) and (3), it follows that $\Delta E_{A B}^{\mathrm{cp}}>\Delta E_{A B}^{\mathrm{uc}}$.

The only meaningful reference value for $\Delta E_{A B}$ for a given wave function model is the CBS limit, for which the BSSE becomes zero. Let us assume that the interaction is attractive, i.e., $\Delta E_{A B}^{\mathrm{cbs}}<0$. In a finite basis, it is usually (but not always ${ }^{16}$ ) observed that the uncorrected interaction energy is too negative compared to $\Delta E_{A B}^{\mathrm{cbs}}$, while the CP-corrected interaction energy is not negative enough,

$$
\Delta E_{A B}^{\mathrm{uc}}<\Delta E_{A B}^{\mathrm{cbs}}<\Delta E_{A B}^{\mathrm{cp}} \quad \text { (common situation). }
$$

An example of this is the MP2/aug-cc-pVTZ interaction energy of the water dimer (detailed in Sec. V), for which $\Delta E_{A B}^{\mathrm{uc}}$ $=-8.14 \mathrm{~m} E_{h}$ and $\Delta E_{A B}^{\mathrm{cp}}=-7.38 \mathrm{~m} E_{h}$, while the CBS estimate is $\Delta E_{A B}^{\mathrm{cbs}}=-7.80 \mathrm{~m} E_{h}$. Thus, the $\mathrm{CP}$ correction changes $\Delta E_{A B}^{\mathrm{uc}}$ towards the CBS result, but the change is too large. It is also worth noting that calculations of interaction energies rely on a very delicate cancellation of basis set incompleteness errors for the total energies. For example, the estimated basis set incompleteness error for the total MP2/aug-cc-pVTZ water dimer energy in Sec. V $\left(E_{A B}^{\text {aug-cc-pVTZ }}-E_{A B}^{\mathrm{cbs}}\right)$ is $71 \mathrm{~m} E_{h}$, which is an order of magnitude larger than the CBS interaction energy $\left(7.80 \mathrm{~m} E_{h}\right)$ !

The above example illustrates that the determination of interaction energies is very sensitive to basis set effects and relies on large error cancellations. For realistically sized basis sets, it is therefore essential that there exists an intricate balance between the description of the compound system $A B$ and the system of noninteracting monomers $A+B$. In Sec. III, we describe how this may be achieved by enforcing that the systems $A B$ and $A+B$ are described in terms of electronic structure calculations that use the same number of optimized wave function parameters.

\section{THE SNOOP SCHEME}

In this work, we focus on $\mathrm{CC}$ interaction energies for closed-shell systems. A CC calculation consists of an initial HF calculation followed by a correlated calculation, and the $\mathrm{CC}$ energy may be written as the sum of the HF and correlation 
components

$$
E=E_{\mathrm{HF}}+E_{\mathrm{corr}} \text {. }
$$

The interaction energy in Eq. (1) can therefore be written as

$$
\Delta E_{A B}=\Delta E_{A B, \mathrm{HF}}+\Delta E_{A B, \text { corr }}
$$

The optimization of the HF wave function may be parameterized as ${ }^{29}$

$$
|\mathrm{HF}(\boldsymbol{\kappa})\rangle=\exp (\hat{\kappa})|\mathrm{HF}\rangle,
$$

where $\hat{\kappa}$ is an anti-Hermitian one-electron operator

$$
\hat{\kappa}=\sum_{a i} \kappa_{i a}\left(E_{i a}-E_{a i}\right) .
$$

In Eq. (9), $\kappa_{i a}$ is an element of the associated anti-Hermitian $\kappa$ rotation matrix, $E_{a i}\left(E_{i a}\right)$ is a singles excitation (de-excitation) operator written in second quantization language, ${ }^{29}$ and $i(a)$ is an occupied (virtual) molecular orbital (MO) index. In the following, $\boldsymbol{\kappa}$ matrices will be denoted as $\boldsymbol{\kappa}_{o, v}$, where $o$ and $v$ are the number of occupied and virtual MOs, respectively. The number of nonredundant parameters in a HF calculation is thus $o v$. A dimer HF calculation is then represented by a matrix $\exp \left(\boldsymbol{\kappa}_{o_{A}+o_{B}, v_{A}+v_{B}}\right)$, where $o_{A}$ and $o_{B}\left(v_{A}\right.$ and $\left.v_{B}\right)$ are the number of occupied (virtual) MOs in monomers $A$ and $B$, respectively, and the number of parameters $P$ in the dimer HF calculation is given by

$$
\begin{aligned}
P_{A B, \mathrm{HF}} & =\left(o_{A}+o_{B}\right)\left(v_{A}+v_{B}\right) \\
& =o_{A}\left(v_{A}+v_{B}\right)+o_{B}\left(v_{A}+v_{B}\right) .
\end{aligned}
$$

Figure 1 illustrates the HF optimizations for the interacting $A B$ dimer and for the uncorrected and $\mathrm{CP}$ monomer calculations. We use calligraphic letters to denote orbital spaces (e.g., $O_{A}$ ), while small Roman letters (e.g., $o_{A}$ ) are used for denoting the corresponding dimensions. Figure 1 shows that the sum of the number of $\kappa$ parameters in the uncorrected/CPcorrected HF monomer calculations are smaller/larger than the number of parameters in the dimer calculation, i.e.,

$$
P_{A+B, \mathrm{HF}}^{\mathrm{uc}}=o_{A} v_{A}+o_{B} v_{B}<P_{A B, \mathrm{HF}}
$$

and

$$
\begin{aligned}
P_{A+B, \mathrm{HF}}^{\mathrm{cp}}= & o_{A}\left(v_{A}+v_{B}+o_{B}\right) \\
& +o_{B}\left(v_{A}+v_{B}+o_{A}\right)>P_{A B, \mathrm{HF}} .
\end{aligned}
$$

Equations (11) and (12) refer to the number of HF parameters, but similar equations can be derived for the number of $\mathrm{CC}$ amplitudes, see Appendix A. One interpretation of Eqs. (11) and (12) is that the variational freedom in the uncorrected/CPcorrected monomer calculations is smaller/larger than in the dimer calculation. For a finite basis set, the uncorrected/CP monomer energies therefore tend to be too high/low compared to the dimer energy, which explains the commonly observed ordering of interaction energies in Eq. (5).

We propose that a better balance between the noninteracting $A+B$ and the interacting $A B$ calculations is obtained if we enforce that the number of optimized parameters in the $A+B$ calculations be equal to the number of optimized parameters in
Interacting AB dimer

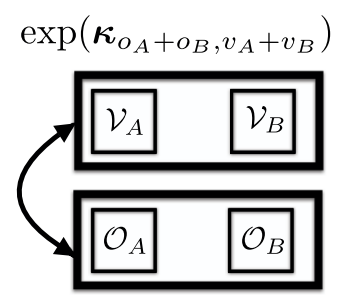

Uncorrected, monomer A

$\exp \left(\boldsymbol{\kappa}_{O_{A}, v_{A}}\right)$

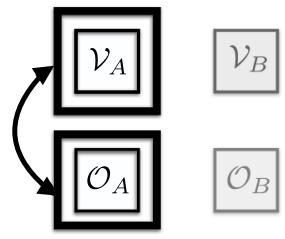

Counterpoise, monomer A

$\exp \left(\boldsymbol{\kappa}_{o_{A}, v_{A}+v_{B}+o_{B}}\right)$

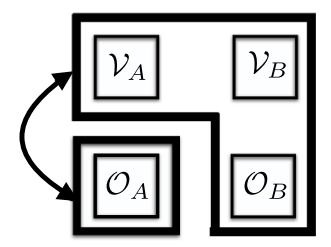

SNOOP, monomer A

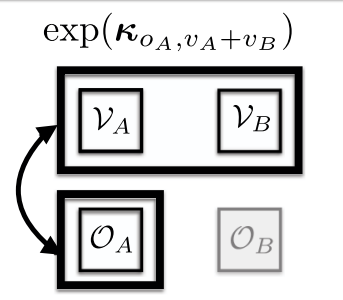

Uncorrected, monomer B

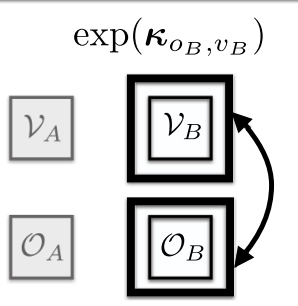

Counterpoise, monomer B $\exp \left(\boldsymbol{\kappa}_{O_{B}, v_{A}+v_{B}+o_{A}}\right)$

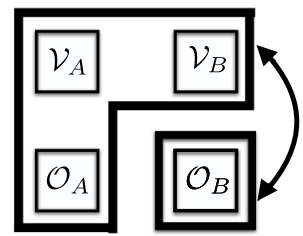

SNOOP, monomer B

$\exp \left(\boldsymbol{\kappa}_{O_{B}, v_{A}+v_{B}}\right)$

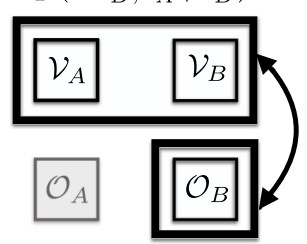

FIG. 1. Schematic illustration of the HF optimizations for the interacting $A B$ dimer and the noninteracting $A+B$ monomers using the uncorrected, $\mathrm{CP}$, and SNOOP approaches. The dimensions of the $\boldsymbol{\kappa}$ rotation matrix are indicated as subscripts. $O_{A}$ and $\mathcal{V}_{A}\left(O_{B}\right.$ and $\left.\mathcal{V}_{B}\right)$ refer to occupied and virtual orbital spaces for monomer $A(B)$. The arrows denote HF orbital optimizations, and grey-shaded orbital spaces are not included in the HF optimizations.

the $A B$ calculation. From Eq. (10), we see that this is achieved if we choose the virtual space of each monomer calculation to be of dimension $v_{A}+v_{B}$, as illustrated in Figure 1 (bottom). For the resulting SNOOP scheme, it thus follows that

$$
P_{A+B, \mathrm{HF}}^{\mathrm{snoop}}=o_{A}\left(v_{A}+v_{B}\right)+o_{B}\left(v_{A}+v_{B}\right)=P_{A B, \mathrm{HF}} .
$$

The optimized orbital spaces of the SNOOP monomer HF calculations are then used in subsequent correlated calculations.

In Appendix A, we demonstrate that Eqs. (11)-(13) effectively hold also for the $\mathrm{CC}$ amplitudes and thus for all parameters entering the $\mathrm{CC}$ calculations. We may therefore generalize 
Eqs. (11)-(13) collectively as

$$
P_{A+B}^{\mathrm{uc}}<P_{A+B}^{\mathrm{snoop}}=P_{A B}<P_{A+B}^{\mathrm{cp}} .
$$

Since the orbital space of the uncorrected calculation is a subset of the SNOOP calculation (see Figure 1), the variational principle implies that the SNOOP monomer HF energies are lower than the uncorrected monomer HF energies. Similarly, the SNOOP monomer orbital space is a subset of the corresponding $\mathrm{CP}$ orbital space, and the $\mathrm{CP}$-corrected monomer $\mathrm{HF}$ energies are therefore lower than the corresponding SNOOP monomer energies. Although these arguments are only strictly true for the variational HF energies, the same features are also observed for the nonvariational CC correlation contributions. We thus conclude that

$$
E_{A}^{\mathrm{uc}}+E_{B}^{\mathrm{uc}} \geq E_{A}^{\mathrm{snoop}}+E_{B}^{\mathrm{snoop}} \geq E_{A}^{\mathrm{cp}}+E_{B}^{\mathrm{cp}},
$$

where the equality signs hold in the CBS limit. Since the same dimer energy $E_{A B}$ is used in the three approaches, it follows from Eq. (1) that

$$
\Delta E_{A B}^{\mathrm{uc}} \leq \Delta E_{A B}^{\mathrm{snoop}} \leq \Delta E_{A B}^{\mathrm{cp}} .
$$

Equations (15) and (16) are summarized in Figure 2 for the case where $\Delta E_{A B}<0$. For the common situation in Eq. (5), in which the uncorrected interaction energies are too attractive, while the CP-corrected interaction energies are not attractive enough, Figure 2 indicates that the SNOOP approach might provide a better balance between the $A+B$ and the $A B$ calculations.

A detailed description of the SNOOP algorithm is given in Figure 3. The orthogonalization of the $\mathcal{V}_{B}$ orbitals in step 2 is not mandatory from a technical point of view, but it simplifies the discussion below. As the basis set of monomer A approaches completeness, linear dependencies might occur when the $\mathcal{V}_{B}^{\perp}$ orbitals are added to the already considered set of orbitals, $\left\{O_{A}, \mathcal{V}_{A}\right\}$. In practice, the linearly independent $\mathcal{V}_{B}^{\perp}$ set of orbitals are obtained by a Gram-Schmidt procedure. If one or more of the orbitals are removed when $\mathcal{V}_{B}^{\perp}$ is formed, the true dimension of the SNOOP virtual space will be smaller than $v_{A}+v_{B}$. In that case, the linear dependency will also be present in the dimer calculation, and the parameter balance between

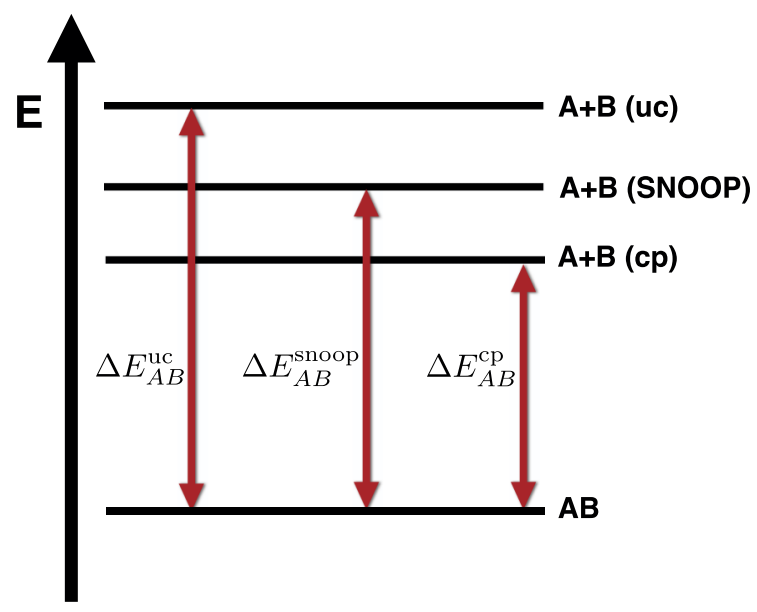

FIG. 2. Energy diagram illustrating the relative positions of uncorrected, SNOOP, and CP noninteracting monomer $A+B$ energies for the situation where $\Delta E_{A B}<0$.
1. Carry out uncorrected HF optimizations for systems $A$ and B to obtain occupied and virtual spaces $O_{A}$ and $\mathcal{V}_{A}$ for monomer $A$, and $O_{B}$ and $\mathcal{V}_{B}$ for monomer $B$.

2. For system A, orthogonalize $\mathcal{V}_{B}$ orbitals against the $O_{A}$ and $\mathcal{V}_{A}$ orbitals to generate orthogonalized $\mathcal{V}_{B}^{\perp}$ orbitals, and carry out $\mathrm{HF}$ optimization in the $\left\{O_{A}, \mathcal{V}_{A}, \mathcal{V}_{B}^{\perp}\right\}$ space to calculate $E_{A, \mathrm{HF}}^{\text {snoop }}$ :

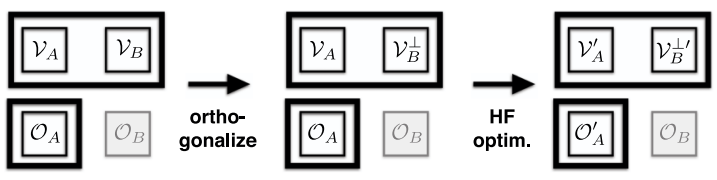

3. Carry out the correlated calculation for system A using optimized occupied $O_{A}^{\prime}$ and virtual $\mathcal{V}_{A}^{\prime} \cup \mathcal{V}_{B}^{\perp \prime}$ orbitals to determine $E_{A, \text { corr }}^{\text {snop }}$

4. Calculate the SNOOP CC energy for monomer A:

$$
E_{A}^{\text {snoop }}=E_{A, \mathrm{HF}}^{\text {snoop }}+E_{A, \text { corr }}^{\text {snoop }} \text {. }
$$

5. Carry out the equivalents of steps 2,3 , and 4 for system $B$.

6. Carry out conventional $A B$ dimer calculation (HF+correlation) in the total orbital space to determine the dimer energy $E_{A B}$.

7. Calculate the SNOOP interaction energy:

$$
\Delta E_{A B}^{\text {snoop }}=E_{A B}-E_{A}^{\text {snoop }}-E_{B}^{\text {snoop }} .
$$

FIG. 3. The SNOOP CC algorithm.

the monomer and dimer calculations described above will still hold. However, for all molecules and basis sets considered in this study (see Sec. V), no orbitals have been removed when forming $\mathcal{V}_{B}^{\perp}$. We also note that the occurrence of linear dependencies in a SNOOP calculation would imply linear dependencies in the corresponding CP calculation since the SNOOP optimization space is a subset of the CP optimization space (see Figure 1).

The SNOOP scheme bears some resemblance to the VCP scheme $^{3,4}$ since the dimension of the virtual space in the correlated monomer calculations is effectively $v_{A}+v_{B}$ in both schemes. However, the orbitals are HF-optimized in the SNOOP scheme (step 2 in Figure 3), while the VCP scheme may be interpreted as omitting this step. The number of HF parameters in a VCP calculation is thus the same as for the uncorrected calculation, and the VCP HF monomer energies are identical to the uncorrected HF monomer energies. The parameter balance of the SNOOP scheme is therefore not present in the VCP scheme at the HF level (and consequently $\left.\Delta E_{A B, \mathrm{HF}}^{\mathrm{snoop}} \neq \Delta E_{A B, \mathrm{HF}}^{\mathrm{vcp}}\right)$. The number of parameters in the correlated calculation is the same for the two approaches, but the correlation contribution to the interaction energy will also be different because the SNOOP orbitals have been optimized in the larger orbital space $\left(\Delta E_{A B \text {, corr }}^{\text {snoop }} \neq \Delta E_{A B \text {, corr }}^{\mathrm{vcp}}\right)$.

\section{COMPUTATIONAL DETAILS}

We consider a test set consisting of the 8 molecular dimers in Figure 4, i.e., the water dimer, the hydrogen fluoride dimer, the formamide dimer, the methane dimer, the benzene-hydrogen sulfide complex, and the sandwich (S), T-shaped, and parallel displaced (PD) conformations of the 

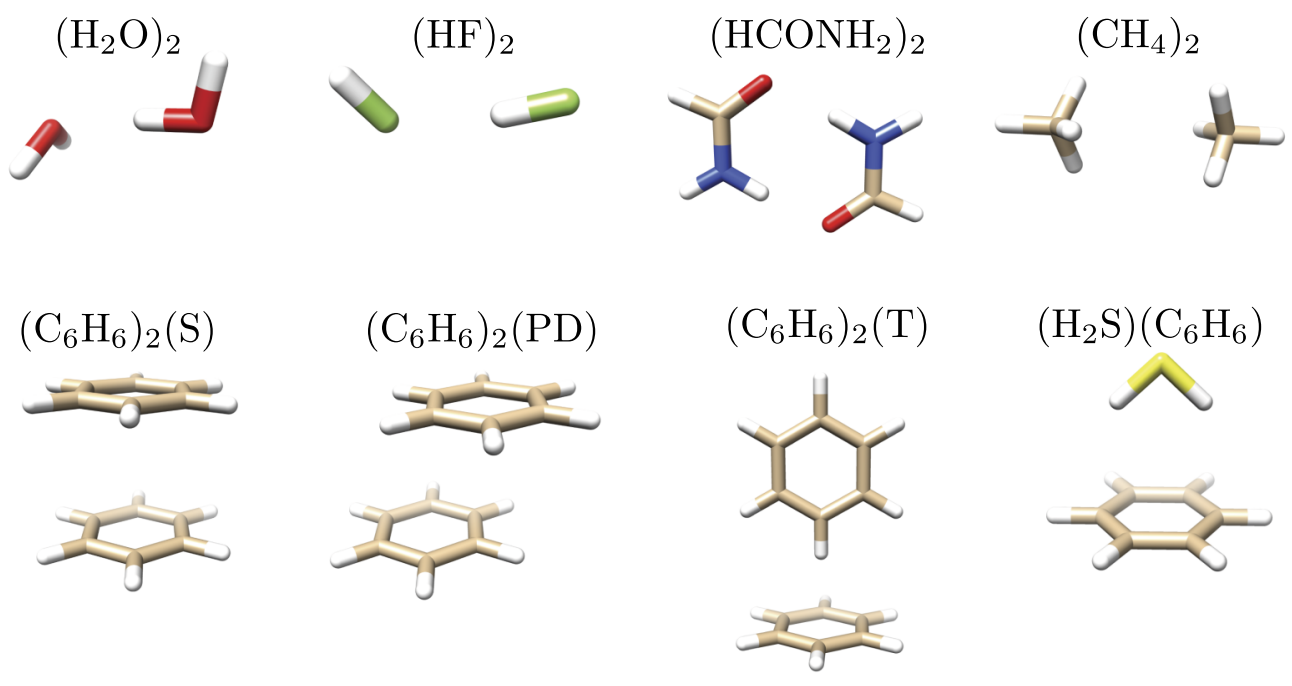

FIG. 4. Molecular dimers considered in this study.

benzene dimer. This test set ranges from molecular systems where the interactions are dominated by hydrogen bonds (e.g., the water dimer) to systems where dispersion forces are dominating (e.g., the methane dimer).

All geometries are equilibrium structures, and the monomer geometries are the same for the isolated monomers and for the dimer complexes. The reference interaction energies are presented in Table I. For details on the molecular geometries and reference interaction energies, cf. Appendix B.

We have carried out MP2 and CCSD(T) calculations using the aug-cc-pVXZ $(\mathrm{X}=\mathrm{D}, \mathrm{T}, \mathrm{Q})$ basis sets. ${ }^{30}$ The HF gradient norm was converged to $10^{-6}$ a.u., and the CCSD residual norm was converged to $10^{-9}$ a.u. All calculations used the frozen core approximation and were carried out using a local version of the LSDALTON program. ${ }^{31}$ For the complexes containing benzene, the diffuse functions were omitted on hydrogen atoms. The CCSD(T)/aug-cc-pVQZ calculations were too large to be carried out for the benzene dimers with our present code (30 occupied and 1278 virtual orbitals).

\section{RESULTS}

\section{A. Comparison of the uncorrected, CP-corrected, and SNOOP schemes}

For a given basis set, we consider the relative error of the interaction energy, $\Delta$,

TABLE I. Estimated MP2 and CCSD(T) CBS interaction energies $\left(\mathrm{m} E_{h}\right)$ for the molecular dimers in Figure 4.

\begin{tabular}{lcc}
\hline \hline System & MP2 & CCSD(T) \\
\hline$\left(\mathrm{H}_{2} \mathrm{O}\right)_{2}$ & -7.80 & -7.91 \\
$(\mathrm{HF})_{2}$ & -7.15 & -7.35 \\
$\left(\mathrm{HCONH}_{2}\right)_{2}$ & -25.1 & -25.4 \\
$\left(\mathrm{CH}_{4}\right)_{2}$ & -0.80 & -0.86 \\
$\left(\mathrm{C}_{6} \mathrm{H}_{6}\right)_{2}(\mathrm{~S})$ & -5.50 & -2.63 \\
$\left(\mathrm{C}_{6} \mathrm{H}_{6}\right)_{2}(\mathrm{PD})$ & -7.82 & -4.25 \\
$\left(\mathrm{C}_{6} \mathrm{H}_{6}\right)_{2}(\mathrm{~T})$ & -5.77 & -4.29 \\
$\left(\mathrm{H}_{2} \mathrm{~S}\right)\left(\mathrm{C}_{6} \mathrm{H}_{6}\right)$ & -5.76 & -4.51 \\
\hline \hline
\end{tabular}

$$
\Delta=\frac{\Delta E^{\text {method }}-\Delta E^{\mathrm{cbs}}}{\left|\Delta E^{\mathrm{cbs}}\right|} \cdot 100 \%,
$$

where $\Delta E^{\text {method }}$ is either the uncorrected (Eq. (2)), the CPcorrected (Eq. (3)), or the SNOOP (Eq. (18)) interaction energy, and $\Delta E^{\mathrm{cbs}}$ is the interaction energy at the estimated CBS limit (Table I). For the MP2 and CCSD(T) models, $\Delta E^{\mathrm{cbs}}<0$ for all molecules in Figure 4, and a negative (positive) $\Delta$ thus implies that the calculated interaction energy is too attractive (not attractive enough). We also consider the mean error, $\bar{\Delta}$, the absolute mean error, $\bar{\Delta}_{\text {abs }}$, the standard deviation, $\sigma$, and the maximum absolute error, $\Delta_{\max }$. The statistical error measures are presented in Tables II and III, respectively, while the relative MP2 and CCSD(T) errors for the individual molecular systems are given in Tables IV and V, respectively. The normal distribution of the MP2 errors is plotted in Figure 5.

With the exception of some of the $\mathrm{CP} \sigma$ values, the statistical measures in Tables II and III are significantly smaller for the SNOOP scheme than for the two other schemes for both the MP2 and CCSD(T) models and regardless of the basis set, see also Figure 5. These findings confirm the theoretical prediction in Sec. III that it is important to use a balanced number of parameters in the monomer and dimer calculations.

TABLE II. Statistical errors for MP2 interaction energies (\%) for the set of test molecules.

\begin{tabular}{lccrc}
\hline \hline Measure & Basis & Uncorrected & \multicolumn{1}{c}{ CP } & SNOOP \\
\hline $\bar{\Delta}$ & aug-cc-pVDZ & -30.0 & 16.6 & 4.4 \\
& aug-cc-pVTZ & -13.1 & 6.6 & 1.2 \\
& aug-cc-pVQZ & -5.2 & 2.7 & 1.1 \\
$\bar{\Delta}_{\text {abs }}$ & aug-cc-pVDZ & 30.0 & 16.6 & 4.5 \\
& aug-cc-pVTZ & 13.1 & 6.6 & 1.8 \\
& aug-cc-pVQZ & 5.2 & 2.7 & 1.1 \\
$\sigma$ & aug-cc-pVDZ & 30.4 & 4.3 & 4.2 \\
& aug-cc-pVTZ & 9.5 & 1.1 & 2.0 \\
& aug-cc-pVQZ & 3.0 & 0.6 & 0.7 \\
& aug-cc-pVDZ & 83.8 & 21.7 & 11.3 \\
$\Delta_{\text {max }}$ & aug-cc-pVTZ & 25.1 & 8.0 & 3.4 \\
& aug-cc-pVQZ & 8.6 & 3.6 & 2.4 \\
\hline \hline
\end{tabular}


TABLE III. Statistical errors for CCSD(T) interaction energies (\%) for the set of test molecules.

\begin{tabular}{lccrc}
\hline \hline Measure & Basis & Uncorrected & \multicolumn{1}{c}{ CP } & SNOOP \\
\hline $\bar{\Delta}$ & aug-cc-pVDZ & -40.2 & 17.7 & 2.1 \\
& aug-cc-pVTZ & -17.7 & 6.5 & 0.1 \\
$\bar{\Delta}_{\text {abs }}$ & aug-cc-pVDZ & 40.2 & 17.7 & 7.1 \\
& aug-cc-pVTZ & 17.7 & 6.5 & 3.1 \\
$\sigma$ & aug-cc-pVDZ & 38.9 & 4.4 & 8.3 \\
& aug-cc-pVTZ & 16.4 & 1.8 & 3.8 \\
& aug-cc-pVDZ & 85.1 & 25.0 & 13.6 \\
$\Delta_{\text {max }}$ & aug-cc-pVTZ & 46.8 & 10.4 & 5.4 \\
& & & & \\
\hline \hline
\end{tabular}

The relatively small $\sigma$ values for the $\mathrm{CP}$ scheme in Tables II and III show that the $\mathrm{CP}$ errors are quite systematic (see Sec. V B).

Tables IV and V collectively show that the uncorrected approach yields interaction energies that are too attractive $(\Delta<0)$ for all dimers of the test set, while the corresponding $\mathrm{CP}$ values are not attractive enough $(\Delta>0)$, in agreement with Eq. (5). The SNOOP errors for the individual molecules are in all cases-with the exception of $\left(\mathrm{C}_{6} \mathrm{H}_{6}\right)_{2}$ (S)/aug-ccpVTZ-smaller than the corresponding CP errors. Smaller errors are in some cases observed for the uncorrected approach, but this approach suffers from large errors for, e.g., the benzene and methane dimers, illustrating that the uncorrected interaction energies are quite unreliable. In accordance with the monomer energy ordering in Figure 2, the SNOOP scheme always yields errors that are sandwiched between the uncor-

TABLE IV. Relative errors $\Delta(\%)$ of uncorrected, CP-corrected, and SNOOP MP2 interaction energies for the test molecules.

\begin{tabular}{|c|c|c|c|c|}
\hline System & Basis & Uncorrected & $\mathrm{CP}$ & SNOOP \\
\hline \multirow[t]{3}{*}{$\left(\mathrm{H}_{2} \mathrm{O}\right)_{2}$} & aug-cc-pVDZ & -5.3 & 12.2 & 6.6 \\
\hline & aug-cc-pVTZ & -4.3 & 5.5 & 2.6 \\
\hline & aug-cc-pVQZ & -2.9 & 2.0 & 1.1 \\
\hline \multirow[t]{3}{*}{$(\mathrm{HF})_{2}$} & aug-cc-pVDZ & -3.1 & 11.4 & 7.0 \\
\hline & aug-cc-pVTZ & -4.5 & 5.8 & 2.7 \\
\hline & aug-cc-pVQZ & -3.7 & 1.8 & 0.7 \\
\hline \multirow[t]{3}{*}{$\left(\mathrm{HCONH}_{2}\right)_{2}$} & aug-cc-pVDZ & -1.9 & 12.0 & 7.2 \\
\hline & aug-cc-pVTZ & -2.6 & 5.2 & 3.4 \\
\hline & aug-cc-pVQZ & -1.4 & 2.3 & 1.8 \\
\hline \multirow[t]{3}{*}{$\left(\mathrm{H}_{2} \mathrm{~S}\right)\left(\mathrm{C}_{6} \mathrm{H}_{6}\right)$} & aug-cc-pVDZ & -5.2 & 19.8 & 11.3 \\
\hline & aug-cc-pVTZ & -6.0 & 8.0 & 3.4 \\
\hline & aug-cc-pVQZ & -2.1 & 3.6 & 2.4 \\
\hline \multirow[t]{3}{*}{$\left(\mathrm{C}_{6} \mathrm{H}_{6}\right)_{2}(\mathrm{~S})$} & aug-cc-pVDZ & -47.4 & 18.0 & 2.6 \\
\hline & aug-cc-pVTZ & -25.1 & 6.9 & -0.4 \\
\hline & aug-cc-pVQZ & -8.3 & 3.1 & 0.5 \\
\hline \multirow[t]{3}{*}{$\left(\mathrm{C}_{6} \mathrm{H}_{6}\right)_{2}(\mathrm{~T})$} & aug-cc-pVDZ & -46.2 & 21.4 & 1.1 \\
\hline & aug-cc-pVTZ & -20.1 & 7.9 & -0.1 \\
\hline & aug-cc-pVQZ & -7.2 & 3.4 & 1.4 \\
\hline \multirow[t]{3}{*}{$\left(\mathrm{C}_{6} \mathrm{H}_{6}\right)_{2}(\mathrm{PD})$} & aug-cc-pVDZ & -46.8 & 16.3 & -0.3 \\
\hline & aug-cc-pVTZ & -20.9 & 6.5 & -1.2 \\
\hline & aug-cc-pVQZ & -7.2 & 2.9 & 0.9 \\
\hline \multirow[t]{3}{*}{$\left(\mathrm{CH}_{4}\right)_{2}$} & aug-cc-pVDZ & -83.8 & 21.7 & -0.2 \\
\hline & aug-cc-pVTZ & -21.1 & 6.9 & -0.5 \\
\hline & aug-cc-pVQZ & -8.6 & 2.7 & 0.4 \\
\hline
\end{tabular}

TABLE V. Relative errors $\Delta(\%)$ of uncorrected, CP-corrected, and SNOOP $\operatorname{CCSD}(\mathrm{T})$ interaction energies for the test molecules.

\begin{tabular}{lccrr}
\hline \hline System & Basis & Uncorrected & CP & SNOOP \\
\hline$\left(\mathrm{H}_{2} \mathrm{O}\right)_{2}$ & aug-cc-pVDZ & -4.8 & 13.9 & 7.8 \\
& aug-cc-pVTZ & -3.9 & 5.8 & 2.9 \\
& aug-cc-pVQZ & -1.9 & 2.1 & 1.4 \\
$(\mathrm{HF})_{2}$ & aug-cc-pVDZ & -3.0 & 13.2 & 8.1 \\
& aug-cc-pVTZ & -4.3 & 6.4 & 3.0 \\
& aug-cc-pVQZ & -2.8 & 2.1 & 1.1 \\
$\left(\mathrm{HCONH}_{2}\right)_{2}$ & aug-cc-pVDZ & -2.3 & 12.2 & 7.1 \\
& aug-cc-pVTZ & -3.3 & 4.2 & 2.4 \\
& aug-cc-pVQZ & -1.9 & 1.0 & 0.6 \\
$\left(\mathrm{H}_{2} \mathrm{~S}\right)_{\left(\mathrm{C}_{6} \mathrm{H}_{6}\right)}$ & aug-cc-pVDZ & -8.5 & 25.0 & 13.6 \\
& aug-cc-pVTZ & -7.1 & 10.4 & 4.5 \\
& aug-cc-pVQZ & -0.9 & 4.8 & 3.6 \\
$\left(\mathrm{C}_{6} \mathrm{H}_{6}\right)_{2}(\mathrm{~S})$ & aug-cc-pVDZ & -85.1 & 20.3 & -7.0 \\
& aug-cc-pVTZ & -46.8 & 5.1 & -5.4 \\
$\left(\mathrm{C}_{6} \mathrm{H}_{6}\right)_{2}(\mathrm{~T})$ & aug-cc-pVDZ & -58.1 & 17.5 & -3.2 \\
& aug-cc-pVTZ & -22.4 & 7.1 & -1.5 \\
$\left(\mathrm{C}_{6} \mathrm{H}_{6}\right)_{2}(\mathrm{PD})$ & aug-cc-pVDZ & -83.6 & 18.5 & -9.6 \\
& aug-cc-pVTZ & -35.6 & 6.8 & -5.1 \\
$\left(\mathrm{CH}_{4}\right)_{2}$ & aug-cc-pVDZ & -76.4 & 20.6 & -0.4 \\
& aug-cc-pVTZ & -18.2 & 6.2 & -0.3 \\
& aug-cc-pVQZ & -5.4 & 2.6 & 1.0 \\
\hline \hline
\end{tabular}

rected and CP-corrected errors, which is why the SNOOP in general leads to smaller errors than the uncorrected and $\mathrm{CP}$ approaches. These findings in connection with Figure 2 also explain why an empirical scaling of the $\mathrm{CP}$ correction in many cases gives improved results over the standard $\mathrm{CP}$ approach. ${ }^{17-19}$ Both positive and negative SNOOP errors are observed. We elaborate on this issue in Sec. V B.

\section{B. Convergence with basis set and extrapolation}

In this section, we analyze the convergence of the SNOOP interaction energies with respect to basis set. This analysis will lead us to conclude that the SNOOP scheme is not suitable for

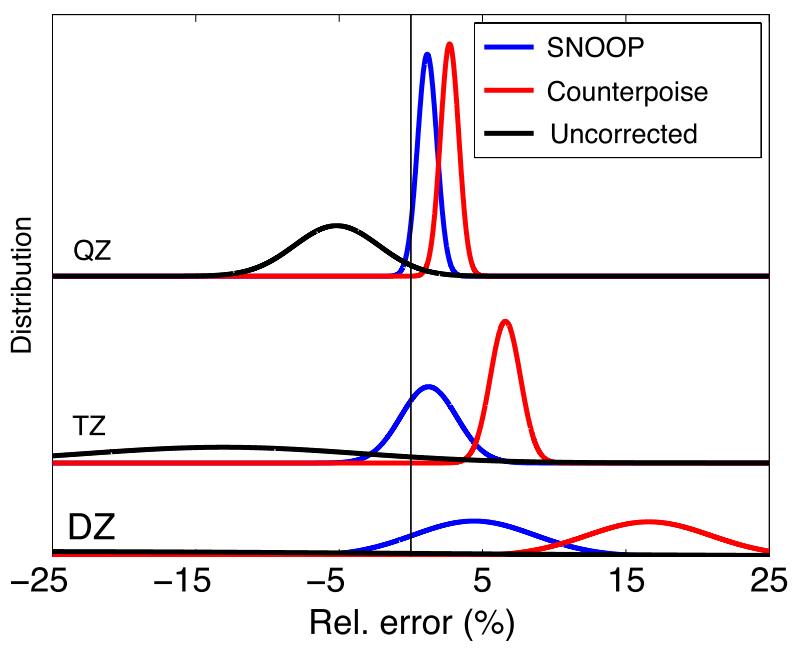

FIG. 5. Normal distributions of the MP2 errors for uncorrected, CPcorrected, and SNOOP interaction energies for the aug-cc-pVDZ (bottom), aug-cc-pVTZ (middle), and aug-cc-pVQZ (top) basis sets. 
use in extrapolation schemes, such as the $X^{-3}$ extrapolation scheme $^{32}$ of Halkier et al. However, we also demonstrate that SNOOP interaction energies obtained using a given basis are of similar quality as those determined by basis set extrapolation of $\mathrm{CP}$ results obtained at a similar computational cost.

To analyze the components of the interaction energy in greater detail, we separate the total interaction energy errors into $\mathrm{HF}$ and correlation contributions. For brevity, we limit the analysis to the water and the PD benzene dimers as representative examples of molecular complexes dominated by hydrogen bonding and dispersion interactions, respectively. Furthermore, we consider only MP2 results since the CCSD(T) results are similar.

The results are presented in Table VI. We recognize that the correlation errors dominate over the HF errors in most cases, and the general superiority of the SNOOP scheme over the uncorrected and CP approaches (Sec. V A) is caused primarily by the comparatively small errors in the correlation part of the SNOOP interaction energy. In addition, the extraordinarily small SNOOP error for the PD benzene dimer using the aug-cc-pVDZ basis is seen to result in part from a fortuitous cancellation of a negative HF error and a positive correlation error.

From Table VI it is clear that the SNOOP interaction energies oscillate slightly when the basis set is increased, as illustrated by the convergence of the $\mathrm{HF}$ contribution for $\left(\mathrm{H}_{2} \mathrm{O}\right)_{2}$ and the correlation contribution for $\left(\mathrm{C}_{6} \mathrm{H}_{6}\right)_{2}(\mathrm{PD})$. On the other hand, the $\mathrm{CP}$ interaction energies display a stable convergence with basis set for the HF as well as the correlation contribution. $\mathrm{CP}$ interaction energies obtained with basis sets of increasing cardinal numbers can therefore be used to extrapolate the interaction energy to the CBS limit, ${ }^{32}$ while such extrapolations would be quite unstable for the SNOOP scheme.

To understand the reason for the oscillatory behavior in the basis set convergence, let us consider the SNOOP scheme in Figure 3. In general, the SNOOP optimization space for mono- mer A, i.e., $\mathcal{S}_{A}=\left\{O_{A}, \mathcal{V}_{A}, \mathcal{V}_{B}^{\perp}\right\}$, contains some components of the $O_{B}$ space. In the following discussion, we formally write the $O_{B}$ space as $O_{B}=O_{B}^{\|} \oplus O_{B}^{\perp}$, where $O_{B}^{\|}$is the component contained in $\mathcal{S}_{A}$, while $O_{B}^{\perp}$ is the orthogonal complement. The SNOOP optimization space will thus effectively include $O_{B}^{\|}$, but not $O_{B}^{\perp}$. As the cardinal number of the basis set is increased, the $O_{B}^{\perp}$ component will become smaller, and it vanishes in the formal limit where the basis of monomer $\mathrm{A},\left\{O_{A}, \mathcal{V}_{A}\right\}$, constitutes a complete set (of course $\mathcal{V}_{B}^{\perp}$ will also vanish in this limit). To quantify the discussion, let us define a quantity, $\xi\left(O_{B}^{\perp}\right)$, which is a measure of the "average amount of $O_{B}^{\perp}$,"

$$
\begin{gathered}
\left|\phi_{i}^{O_{B}^{\perp}}\right\rangle=\left(\hat{1}-\hat{P}_{A}\right)\left|\phi_{i}^{O_{B}}\right\rangle, \\
\xi\left(O_{B}^{\perp}\right)=\frac{1}{o_{B}} \sum_{i=1}^{O_{B}}\left\langle\phi_{i}^{O^{\perp}} \mid \phi_{i}^{O^{\perp}}\right\rangle .
\end{gathered}
$$

In Eq. (20), $\hat{P}_{A}$ is a projection operator onto the $\mathcal{S}_{A}$ space, $\left|\phi_{i}^{O_{B}}\right\rangle$ is an occupied orbital for monomer B (optimized using the basis functions on B), and we recall that $o_{B}$ in Eq. (21) is the number of occupied orbitals for monomer $\mathrm{B}$.

In the limit where $\left\{O_{A}, V_{A}\right\}$ forms a complete set, it follows from Eqs. (20) and (21) that $\xi\left(O_{B}^{\perp}\right)=0\left(O_{B}^{\perp}\right.$ is an empty set because $\hat{P}_{A}$ is the identity operator), while $\xi\left(O_{B}^{\perp}\right)=1$ in the other limit where $O_{B}=O_{B}^{\perp}$ contains no components of $\mathcal{S}_{A} \cdot \xi\left(O_{B}^{\perp}\right)$ is thus a measure of the fraction of the $O_{B}$ space which is not considered in the optimization space for monomer A. $\xi\left(O_{A}^{\perp}\right)$ may be defined analogously. The $\xi\left(O_{A}^{\perp}\right)$ and $\xi\left(O_{B}^{\perp}\right)$ measures for the water dimer and the PD benzene dimer are given in Table VII. As expected, Table VII shows that $\xi\left(O_{B}^{\perp}\right)$ and $\xi\left(O_{A}^{\perp}\right)$ decrease when the cardinal number is increased and the basis sets on $\mathrm{A}$ and $\mathrm{B}$ each approaches completeness. The convergence of $\xi\left(O_{A}^{\perp}\right)$ and $\xi\left(O_{B}^{\perp}\right)$ towards 0 is a measure of the convergence towards completeness for the aug-cc-pVXZ family of basis set.

TABLE VI. Relative interaction energy errors $\Delta(\%)$ for uncorrected, CP-corrected, and SNOOP MP2 interaction

\begin{tabular}{|c|c|c|c|c|c|}
\hline System & Method & Basis & Hartree-Fock & Correlation & Total \\
\hline \multirow[t]{9}{*}{$\left(\mathrm{H}_{2} \mathrm{O}\right)_{2}$} & \multirow[t]{3}{*}{ Uncorrected } & aug-cc-pVDZ & -4.6 & -0.7 & -5.3 \\
\hline & & aug-cc-pVTZ & -0.8 & -3.5 & -4.3 \\
\hline & & aug-cc-pVQZ & -0.6 & -2.2 & -2.9 \\
\hline & \multirow[t]{3}{*}{$\mathrm{CP}$} & aug-cc-pVDZ & 0.5 & 11.7 & 12.2 \\
\hline & & aug-cc-pVTZ & 0.8 & 4.7 & 5.5 \\
\hline & & aug-cc-pVQZ & 0.0 & 2.0 & 2.0 \\
\hline & \multirow[t]{3}{*}{ SNOOP } & aug-cc-pVDZ & -0.7 & 7.3 & 6.6 \\
\hline & & aug-cc-pVTZ & 0.5 & 2.2 & 2.6 \\
\hline & & aug-cc-pVQZ & -0.1 & 1.2 & 1.1 \\
\hline \multirow[t]{9}{*}{$\left(\mathrm{C}_{6} \mathrm{H}_{6}\right)_{2}(\mathrm{PD})$} & \multirow[t]{3}{*}{ Uncorrected } & aug-cc-pVDZ & -20.0 & -26.8 & -46.8 \\
\hline & & aug-cc-pVTZ & -4.6 & -16.3 & -20.9 \\
\hline & & aug-cc-pVQZ & -1.2 & -6.0 & -7.2 \\
\hline & \multirow[t]{3}{*}{$\mathrm{CP}$} & aug-cc-pVDZ & -0.1 & 16.4 & 16.3 \\
\hline & & aug-cc-pVTZ & 0.0 & 6.4 & 6.5 \\
\hline & & aug-cc-pVQZ & 0.0 & 2.9 & 2.9 \\
\hline & \multirow[t]{3}{*}{ SNOOP } & aug-cc-pVDZ & -5.8 & 5.5 & -0.3 \\
\hline & & aug-cc-pVTZ & -1.1 & -0.1 & -1.2 \\
\hline & & aug-cc-pVQZ & -0.1 & 1.0 & 0.9 \\
\hline
\end{tabular}
energies partitioned into HF and correlation contributions for the water dimer and the PD benzene dimer. 
TABLE VII. The $\xi\left(O_{A}^{\perp}\right)$ and $\xi\left(O_{B}^{\perp}\right)$ measures for the water and PD benzene dimers. $\xi\left(O_{B}^{\perp}\right)$ is a measure of the fraction of the $O_{B}$ space which is not considered in the optimization space for monomer A (see text for details). For the water dimer, subsystem A (B) is depicted to the left (right) in Figure 4. For the PD benzene dimer subsystems $\mathrm{A}$ and $\mathrm{B}$ are equivalent (see Figure 4) and therefore $\xi\left(O_{A}^{\perp}\right)=\xi\left(O_{B}^{\perp}\right)$.

\begin{tabular}{lccc}
\hline \hline System & Basis & $\xi\left(O_{A}^{\perp}\right)$ & $\xi\left(O_{B}^{\perp}\right)$ \\
\hline$\left(\mathrm{H}_{2} \mathrm{O}\right)_{2}$ & aug-cc-pVDZ & 0.69 & 0.75 \\
& aug-cc-pVTZ & 0.41 & 0.59 \\
& aug-cc-pVQZ & 0.22 & 0.38 \\
$\left(\mathrm{C}_{6} \mathrm{H}_{6}\right)_{2}(\mathrm{PD})$ & aug-cc-pVDZ & 0.76 & 0.76 \\
& aug-cc-pVTZ & 0.61 & 0.61 \\
& aug-cc-pVQZ & 0.47 & 0.47 \\
\hline \hline
\end{tabular}

The fact that $\xi\left(O_{A}^{\perp}\right)$ and $\xi\left(O_{B}^{\perp}\right)$ decrease with the size of the basis set implies that variable components of $O_{B}$ will be included in the optimization space for monomer A (and vice versa). The basis set is therefore not systematically improved when the cardinal number is increased, and the convergence of the SNOOP monomer energies with basis set is not as uniform as is the case for the dimer energy. This is the reason for the oscillatory behavior of some of the SNOOP interaction energy components in Table VI. On the other hand, all $O_{B}^{\perp}$ components are considered for all basis sets in the CP scheme (see Figure 1), and the convergence of the $\mathrm{CP}$ interaction energies with basis set is stable. The CP scheme is therefore suited for use in extrapolation schemes (e.g., the $X^{-3}$ extrapolation scheme ${ }^{32}$ ), whereas the SNOOP scheme is not.

Obviously, the small oscillatory behavior of the SNOOP basis set convergence is a downside compared to the $\mathrm{CP}$ scheme. On the other hand, the parameter balance between the monomer and dimer calculations is an attractive feature of the SNOOP scheme, which is not present in the CP scheme. An ideal approach for calculating intermolecular interaction energies would yield a stable convergence with basis set and have a balanced treatment of the parameters in the monomer and dimer calculations. In practice, however, one has to be pragmatic and choose either the parameter balance (SNOOP) or the stable basis set convergence (CP). The results discussed in Sec. V A show that for a given basis set, the SNOOP scheme yields results closer to the CBS limit than the CP scheme, indicating that the parameter balance may indeed be very important.

From a practical point of view, a relevant question is whether the SNOOP interaction energy calculated with a given basis set like aug-cc-pVTZ is closer to the CBS value than the corresponding $\mathrm{CP}$ interaction energy obtained by extrapolating aug-cc-pVDZ and aug-cc-pVTZ results (henceforth referred to as DZ-TZ extrapolation). These two approaches have roughly the same computational cost, since an aug-cc-pVDZ calculation is relatively inexpensive compared to an aug-cc-pVTZ calculation, and since the SNOOP and CP calculations using the aug-cc-pVTZ basis cost roughly the same (the SNOOP calculation is slightly cheaper because the virtual space is marginally smaller, see Figure 1).

Table VIII shows that the SNOOP/aug-cc-pVTZ interaction energies are of similar quality as the extrapolated $\mathrm{CP}$
TABLE VIII. Statistical errors (\%) for SNOOP/aug-cc-pVTZ and extrapolated DZ-TZ CP interaction energies where the latter were obtained using the $X^{-3}$ extrapolation scheme. ${ }^{32}$

\begin{tabular}{lccccc}
\hline \hline Model & Scheme & $\bar{\Delta}$ & $\bar{\Delta}_{\text {abs }}$ & $\sigma$ & $\Delta_{\max }$ \\
\hline MP2 & SNOOP (TZ) & 1.2 & 1.8 & 2.0 & 3.4 \\
& CP (DZ-TZ) & 2.1 & 2.1 & 1.3 & 3.7 \\
CCSD(T) & SNOOP (TZ) & 0.1 & 3.1 & 3.8 & 5.4 \\
& CP (DZ-TZ) & 1.7 & 1.8 & 1.2 & 3.5 \\
\hline \hline
\end{tabular}

interaction energies. The SNOOP approach is therefore an attractive alternative to extrapolating $\mathrm{CP}$ interaction energies. For application purposes, it may be advantageous to use both approaches and compare the results to validate the interaction energy of interest. For example, a large difference between $\mathrm{SNOOP} / \mathrm{TZ}$ results and extrapolated CP/DZ-TZ results would be a strong indication that it is necessary to increase the basis set to obtain a reliable interaction energy.

Finally, we note that most of the reference data in Table I are based on TZ-QZ extrapolations of CP interaction energies. However, a generalization of the results in Table VIII to higher cardinal numbers might indicate that the TZ-QZ extrapolated CP interaction energies in fact contain errors of the same magnitude as the SNOOP/aug-cc-pVQZ results. It is thus possible that the SNOOP/aug-cc-pVQZ errors in Tables IV and $\mathrm{V}$ in reality reflect the accuracy of the reference data and not the inherent accuracy.

\section{CONCLUSION AND PERSPECTIVES}

We have presented the SNOOP scheme for the calculation of intermolecular interaction energies. The main philosophy behind the SNOOP scheme is to enforce that the number of optimized wave function parameters for the noninteracting monomer calculations is the same as for the interacting dimer calculation. This strategy tackles the BSSE problem by ensuring a delicate balance between the monomer and dimer calculations for finite basis sets.

Numerical results for the aug-cc-pVXZ $(X=D, T, Q)$ basis sets show that the SNOOP scheme outperforms the uncorrected and CP approaches for the MP2 and $\operatorname{CCSD}(\mathrm{T})$ models for a selection of test molecules. This may be rationalized from the fact that the SNOOP interaction energies by construction are sandwiched between the uncorrected and CPcorrected interaction energies, which tend to be too attractive and not attractive enough, respectively. The uncorrected, CP, and SNOOP schemes naturally converge to the same result in the limit of a complete basis set for a given wave function model.

The SNOOP scheme displays small oscillations in the convergence of the interaction energy when the basis set is systematically increased. The SNOOP scheme is therefore not suited for use in extrapolation techniques, in contrast to the $\mathrm{CP}$ scheme. On the other hand, the delicate parameter balance between the monomer and dimer calculations is an attractive feature of the SNOOP scheme, which is not present in the CP scheme. The numerical results show that, for a given basis 
set, the SNOOP scheme yields results closer to the CBS limit than the $\mathrm{CP}$ scheme, indicating that the parameter balance is indeed very important for practical calculations. Furthermore, SNOOP interaction energies calculated using a given basis are of similar quality as those determined by basis set extrapolation of $\mathrm{CP}$ results obtained at a similar computational cost. The SNOOP approach is therefore an attractive alternative to extrapolating CP interaction energies. For application purposes, it may be particularly advantageous to use both approaches and compare the results in order to validate the interaction energy of interest.

In the SNOOP scheme, the dimer HF calculation is carried out as a conventional HF calculation using the basis functions of monomers $A$ and $B$. The HF optimization for monomer $A$ may be viewed as a relaxation of the uncorrected occupied $A$ orbitals in the total (uncorrected) virtual space (and similarly for monomer $B$ ). The SNOOP approach is therefore easily generalized to more than two interacting systems by allowing the occupied orbitals for each monomer to relax in the total (uncorrected) virtual space.

The computational cost of the SNOOP scheme is that of the underlying wave function model, e.g., $N^{5}$ and $N^{7}$ for the MP2 and CCSD(T) models considered here $(N$ being a measure of the system size). Future developments include the adaption of the SNOOP philosophy to local correlation methods to allow intermolecular interaction energies for large molecular systems to be determined at a linear-scaling cost.

\section{ACKNOWLEDGMENTS}

The research leading to these results has received funding from the European Research Council under the European Union's Seventh Framework Programme (FP/2007-2013)/ ERC Grant Agreement No. 291371 and The Danish Council for Independent Research-Natural Sciences. This research used resources of the Oak Ridge Leadership Computing Facility at Oak Ridge National Laboratory, which is supported by the Office of Science of the Department of Energy under Contract No. DE-AC05-00OR22725.

\section{APPENDIX A: CC PARAMETER ANALYSIS}

In Sec. III, we demonstrated that the total number of HF parameters in the SNOOP monomer calculations equals the number of HF parameters in the dimer calculation. In this appendix, we demonstrate how this is also true for the number of $\mathrm{CC}$ amplitudes. For this analysis, it is convenient to analyze the SNOOP interaction energy in terms of localized HF orbitals. We note that localized HF orbitals are used only for analytical purposes here, while a practical SNOOP implementation can use any set of optimized HF orbitals.

We consider first the CCSD model and next generalize the results to higher-order CC models. The CCSD correlation energy for a closed-shell system may be written as ${ }^{29}$

$$
E_{\mathrm{corr}}=\sum_{i j a b}\left(t_{a i b j}+t_{a i} t_{b j}\right) L_{a i b j},
$$

where $t_{a i}$ and $t_{a i b j}$ are CCSD singles and doubles amplitudes, respectively, while $L_{a i b j}=2 g_{a i b j}-g_{a j b i}$, where $g_{p q r s}=(p q \mid r s)$ is a two-electron integral written in Mulliken notation. In the following, the indices $i, j$, and $k$ denote occupied orbitals, while $a, b$, and $c$ denote virtual orbitals. The correlation contribution to the interaction energy in Eq. (7), $\Delta E_{A B, \text { corr }}$, may thus be partitioned into a singles contribution (sing) and a doubles contribution (doub),

$$
\begin{aligned}
\Delta E_{A B, \text { corr }}= & \Delta E_{A B, \text { sing }}+\Delta E_{A B, \text { doub }} \\
\Delta E_{A B, \text { sing }}= & \sum_{i j a b} t_{a i}^{A B} t_{b j}^{A B} L_{a i b j}^{A B}-\sum_{i j a b} t_{a i}^{A} t_{b j}^{A} L_{a i b j}^{A} \\
& -\sum_{i j a b} t_{a i}^{B} t_{b j}^{B} L_{a i b j}^{B}, \\
\Delta E_{A B, \text { doub }}= & \sum_{i j a b} t_{a i b j}^{A B} L_{a i b j}^{A B}-\sum_{i j a b} t_{a i b j}^{A} L_{a i b j}^{A} \\
& -\sum_{i j a b} t_{a i b j}^{B} L_{a i b j}^{B},
\end{aligned}
$$

where the amplitudes and integrals have been labelled with $A, B$, and $A B$ superscripts to emphasize the calculation from which they have been determined.

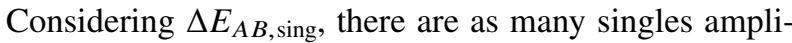
tudes $t_{a i}$ in the correlated $A, B$, and $A B$ calculations as there are $\kappa_{a i}$ parameters in the corresponding HF calculations (see Sec. III), and we therefore directly obtain the analogues of Eqs. (10)-(12) for the singles amplitudes,

$$
\begin{gathered}
P_{A+B, \text { sing }}^{\mathrm{uc}}=o_{A} v_{A}+o_{B} v_{B}<P_{A B, \text { sing }}, \\
P_{A+B, \text { sing }}^{\mathrm{cp}}=o_{A}\left(v_{A}+v_{B}+o_{B}\right)+o_{B}\left(v_{A}+v_{B}+o_{A}\right) \\
>P_{A B, \text { sing }} \\
P_{A+B, \text { sing }}^{\text {snoop }}=P_{A B, \text { sing }}=o_{A}\left(v_{A}+v_{B}\right)+o_{B}\left(v_{A}+v_{B}\right) .
\end{gathered}
$$

To analyze the number of doubles amplitudes, we first partition $\Delta E_{A B \text {, doub }}$ into two contributions where the localized HF orbitals $i$ and $j$ are assigned either to two different monomers, $E_{A B \text {,doubint, }}$ or to the same monomer, $\Delta E_{A B \text {,doubmono, }}$

$$
\begin{gathered}
\Delta E_{A B, \text { doub }}=E_{A B, \text { doubint }}+\Delta E_{A B, \text { doubmono }}, \\
E_{A B, \text { doubint }}=\sum_{i \in A, j \in B} \sum_{a b} t_{a i b j}^{A B} L_{a i b j}^{A B} \\
+\sum_{i \in B, j \in A} \sum_{a b} t_{a i b j}^{A B} L_{a i b j}^{A B}, \\
\Delta E_{A B, \text { doubmono }}=\left(E_{A B, \text { doubmono }}-E_{A, \text { doub }}-E_{B, \text { doub }}\right), \\
E_{A B, \text { doubmono }}=\sum_{i j \in A} \sum_{a b} t_{a i b j}^{A B} L_{a i b j}^{A B} \\
+\sum_{i j \in B} \sum_{a b} t_{a i b j}^{A B} L_{a i b j}^{A B}, \\
E_{A, \text { doub }}=\sum_{i j a b} t_{a i b j}^{A} L_{a i b j}^{A}, \\
E_{B, \text { doub }}=\sum_{i j a b} t_{a i b j}^{B} L_{a i b j}^{B} .
\end{gathered}
$$

Qualitatively, the doubles contribution to the interaction energy may be interpreted in the following manner. $E_{A B \text {, doubint }}$ describes the direct interactions associated with a simultaneous excitation of two localized HF orbitals assigned to the two different monomers, representing dispersion and charge 
transfer effects. On the contrary, the $\Delta E_{A B \text {, doubmono }}$ term involves only $A B$ amplitudes, for which the occupied orbitals are assigned to the same monomer, and $\Delta E_{A B \text {, doubmono thus }}$ describes the indirect relaxation of correlation effects within system $A$ in the presence of $B$ (and vice versa). The type of amplitudes entering $E_{A B \text {, doubint }}$ exists only for the $A B$ dimer, while the type of amplitudes entering $\triangle E_{A B \text {, doubmono }}$ are present also in the monomer calculations. It is therefore reasonable to compare the number of doubles amplitudes in the noninteracting monomer calculations to the number of doubles amplitudes in the $\Delta E_{A B \text {, doubmono }}$ term (and not the $E_{A B \text {, doubint }}$ term). From Eq. (A9), it is clear that the number of amplitudes entering the

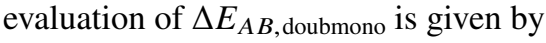

$$
P_{A B, \text { doubmono }}=o_{A}^{2}\left(v_{A}+v_{B}\right)^{2}+o_{B}^{2}\left(v_{A}+v_{B}\right)^{2} .
$$

Considering monomer $A$, there are $o_{A}$ occupied orbitals for all three schemes, while there are $v_{A}, v_{A}+v_{B}+o_{B}$, and $v_{A}+v_{B}$ virtual orbitals for the uncorrected, $\mathrm{CP}$, and SNOOP schemes, respectively, and similarly for monomer $B$ (see Figure 1). We thus find the following number of doubles amplitudes in the various calculations:

$$
\begin{aligned}
P_{A+B, \text { doub }}^{\mathrm{uc}}= & o_{A}^{2} v_{A}^{2}+o_{B}^{2} v_{B}^{2}<P_{A B, \text { doubmono, }} \\
P_{A+B, \text { doub }}^{\mathrm{cp}} & o_{A}^{2}\left(v_{A}+v_{B}+o_{B}\right)^{2}+o_{B}^{2}\left(v_{A}+v_{B}+o_{A}\right)^{2} \\
& >P_{A B, \text { doubmono }} \\
P_{A+B, \text { doub }}^{\text {snoop }} & o_{A}^{2}\left(v_{A}+v_{B}\right)^{2}+o_{B}^{2}\left(v_{A}+v_{B}\right)^{2} \\
= & P_{A B, \text { doubmono. }}
\end{aligned}
$$

We have thus demonstrated that Eq. (14) holds for both the HF parameters (Sec. III), the CCSD singles amplitudes (Eq. (A5)), and the CCSD doubles amplitudes (Eq. (A13)). A prerequisite for this analysis is that only the dimer doubles amplitudes, for which the localized HF occupied orbitals are all assigned to the same monomer, are considered (e.g., $i, j \in A$ or $i, j \in B$ for doubles amplitudes $t_{a i b j}^{A B}$ ). This is reasonable since only these amplitudes have counterparts in the $A+B$ calculations. These conclusions are valid also for higher $\mathrm{CC}$ excitation levels. For example, in the dimer $A B$ calculation, the number of triples amplitudes $t_{a i b j k c}^{A B}$ for which $i, j$, and $k$ are all assigned to monomer $A$ or all assigned to monomer $B$ are $o_{A}^{3}\left(v_{A}+v_{B}\right)^{3}$ and $o_{B}^{3}\left(v_{A}+v_{B}\right)^{3}$, respectively. For the noninteracting monomers in the SNOOP scheme, there are $o_{A}^{3}\left(v_{A}+v_{B}\right)^{3} t_{a i b j k c}^{A}$ amplitudes for monomer $A$ and $o_{B}^{3}\left(v_{A}\right.$ $\left.+v_{B}\right)^{3} t_{a i b j k c}^{B}$ amplitudes for monomer $B$. Hence, the number of $A B$ triples amplitudes, for which $i, j$, and $k$ are assigned to the same monomer, equals the sum of the number of triples amplitudes in the $A+B$ calculations.

\section{APPENDIX B: REFERENCE GEOMETRIES AND INTERACTION ENERGIES}

For the water dimer, we used the CCSD(T)/aug-cc-pVTZ geometry given in Ref. 33. To determine the HF part of the reference interaction energy, $\Delta E_{A B, \mathrm{HF}}$, we simply used the augcc-pVQZ CP-corrected interaction energy. The correlation part, $\Delta E_{A B \text {, corr }}$, was determined by applying the $X^{-3}$ extrapolation scheme ${ }^{32}$ to the CP-corrected aug-cc-pVTZ and augcc-pVQZ interaction energies.
For the hydrogen fluoride dimer, we used the MP2/TZVP geometry optimized for dimer. ${ }^{34}$ The reference interaction energy was determined as for the water dimer.

For the formamide dimer, the geometry and reference interaction energies of Ref. 35 were used.

For $\left(\mathrm{H}_{2} \mathrm{~S}\right)\left(\mathrm{C}_{6} \mathrm{H}_{6}\right)$, the geometry and $\operatorname{CCSD}(\mathrm{T})$ reference interaction energy were taken from Table VI of Ref. 36. The MP2 calculations used the same geometry, but the reference interaction energy was determined as for the water dimer.

For the benzene dimers, we used MP2-optimized geometries for the MP2 calculations and CCSD(T)-optimized geometries for the CCSD(T) calculations. MP2 geometries and reference interaction energies were taken from Tables I and III of Ref. 36, while the CCSD(T) data were taken from Table VI of Ref. 36.

For the methane dimer, the geometry and reference interaction energies were taken from Ref. 36. The methane-methane distance was $3.7 \AA$ for the MP2 as well as for the $\operatorname{CCSD}(\mathrm{T})$ calculations.

${ }^{1}$ B. Liu and A. McLean, J. Chem. Phys. 59, 4557 (1973).

${ }^{2}$ S. F. Boys and F. Bernardi, Mol. Phys. 19, 553 (1970).

${ }^{3}$ J. Daudey, P. Claverie, and J. Malrieu, Int. J. Quantum Chem. 8, 1 (1974).

${ }^{4}$ J. H. van Lenthe, T. van Dam, F. B. van Duijneveldt, and L. M. J. KroonBatenburg, Faraday Symp. Chem. Soc. 19, 125 (1984).

${ }^{5} \mathrm{M}$. Gutowski, J. van Lenthe, J. Verbeek, F. van Duijneveldt, and G. Chałasinski, Chem. Phys. Lett. 124, 370 (1986).

${ }^{6}$ M. Gutowski, F. B. van Duijneveldt, G. Chalasinki, and L. Piela, Mol. Phys. 61, 233 (1987).

${ }^{7}$ G. Chalasinski and M. Gutowski, Chem. Rev. 88, 943 (1988).

${ }^{8}$ J. H. van Lenthe, J. G. C. M. van Duijneveldt-van de Rijdt, and F. B. van Duijneveldt, Adv. Chem. Phys. 69, 521 (1987).

${ }^{9}$ S. Scheiner, Reviews in Computational Chemistry (VCH, New York, 1991), p. 165.

${ }^{10}$ I. Mayer and L. Turi, J. Mol. Struct.: THEOCHEM 227, 43 (1991).

${ }^{11}$ M. Gutowski, J. G. C. M. van Duijneveldt-van de Rijdt, J. H. van Lenthe, and F. B. van Duijneveldt, J. Chem. Phys. 98, 4728 (1993).

${ }^{12}$ M. Gutowski and G. Chalasinski, J. Chem. Phys. 98, 5540 (1993).

${ }^{13}$ D. Cook, J. A. Sordo, and T. L. Sordo, Int. J. Quantum Chem. 48, 375 (1993)

${ }^{14}$ F. B. van Duijneveldt, J. G. C. M. van Duijneveldt-vande Rijdt, and J. H. van Lenthe, Chem. Rev. 94, 1873 (1994).

${ }^{15}$ R. Wieczorek, L. Haskamp, and J. J. Dannenberg, J. Phys. Chem. A 108, 6713 (2004).

${ }^{16}$ L. M. Mentel and E. J. Baerends, J. Chem. Theory Comput. 10, 252 (2013).

${ }^{17}$ M. Schütz, S. Brdarski, P.-O. Widmark, R. Lindh, and G. Karlström, J. Chem. Phys. 107, 4597 (1997).

${ }^{18}$ L. A. Burns, M. S. Marshall, and C. D. Sherrill, J. Chem. Theory Comput. 10, 49 (2014).

${ }^{19}$ B. Brauer, M. K. Kesharwani, and J. M. Martin, J. Chem. Theory Comput. 10, 3791 (2014).

${ }^{20}$ H. Kruse and S. Grimme, J. Chem. Phys. 136, 154101 (2012).

${ }^{21}$ B. Jeziorski and W. Kolos, Molecular Interactions (Wiley, New York, 1982), p. 1.

${ }^{22}$ B. Jeziorski, R. Moszynski, and K. Szalewicz, Chem. Rev. 94, 1887 (1994).

${ }^{23}$ I. Mayer, Int. J. Quantum Chem. 23, 341 (1983).

${ }^{24}$ J. Noga and A. Vibok, Chem. Phys. Lett. 180, 114 (1991).

${ }^{25}$ J. M. Cullen, Int. J. Quantum Chem. Symp. 25, 193 (1991).

${ }^{26}$ A. J. Sadlej, J. Chem. Phys. 95, 6705 (1991).

${ }^{27}$ C. Møller and M. S. Plesset, Phys. Rev. 46, 618 (1934).

${ }^{28}$ K. Raghavachari, G. W. Trucks, J. A. Pople, and M. Head-Gordon, Chem. Phys. Lett. 157, 479 (1989).

${ }^{29}$ T. Helgaker, P. Jørgensen, and J. Olsen, Molecular Electronic Structure Theory, 1st ed. (Wiley, Chichester, England, 2000).

${ }^{30}$ T. H. Dunning, Jr., J. Chem. Phys. 90, 1007 (1989).

${ }^{31}$ K. Aidas, C. Angeli, K. L. Bak, V. Bakken, R. Bast, L. Boman, O. Christiansen, R. Cimiraglia, S. Coriani, P. Dahle, E. K. Dalskov, U. Ekström, T. 
Enevoldsen, J. J. Eriksen, P. Ettenhuber, B. Fernández, L. Ferrighi, H. Fliegl, L. Frediani, K. Hald, A. Halkier, C. Hättig, H. Heiberg, T. Helgaker, A. C. Hennum, H. Hettema, E. Hjertenæs, S. Høst, I.-M. Høyvik, M. F. Iozzi, B. Jansik, H. J. A. Jensen, D. Jonsson, P. Jørgensen, J. Kauczor, S. Kirpekar, T. Kjærgaard, W. Klopper, S. Knecht, R. Kobayashi, H. Koch, J. Kongsted, A. Krapp, K. Kristensen, A. Ligabue, O. B. Lutnæs, J. I. Melo, K. V. Mikkelsen, R. H. Myhre, C. Neiss, C. B. Nielsen, P. Norman, J. Olsen, J. M. H. Olsen, A. Osted, M. J. Packer, F. Pawlowski, T. B. Pedersen, P. F. Provasi, S. Reine, Z. Rinkevicius, T. A. Ruden, K. Ruud, V. Rybkin, P. Salek, C. C. M. Samson, A. S. de Merás, T. Saue, S. P. A. Sauer, B. Schimmelpfennig, K. Sneskov, A. H. Steindal, K. O. Sylvester-Hvid, P. R. Taylor, A. M. Teale, E. I. Tellgren, D. P. Tew, A. J. Thorvaldsen, L. Thøgersen, O. Vahtras, M. A. Watson, D. J.
D. Wilson, M. Ziolkowski, and H. Ågren, WIREs Comput. Mol. Sci. 4, 269 (2013)

${ }^{32}$ A. Halkier, T. Helgaker, P. Jørgensen, W. Klopper, H. Koch, J. Olsen, and A. K. Wilson, Chem. Phys. Lett. 286, 243 (1998).

${ }^{33}$ A. Halkier, H. Koch, P. Jørgensen, O. Christiansen, I. M. B. Nielsen, and T. Helgaker, Theor. Chim. Acta 97, 150 (1997).

${ }^{34} \mathrm{Hydrogen}$ fluroide dimer geometry at the MP2/TZVP level is available at http://cccbdb.nist.gov.

${ }^{35}$ P. Jurečka, J. Šponer, J. Černỳ, and P. Hobza, Phys. Chem. Chem. Phys. 8, 1985 (2006).

${ }^{36}$ C. D. Sherrill, T. Takatani, and E. G. Hohenstein, J. Phys. Chem. A 113, 10146 (2009). 